\title{
Synergistic Integration of Agent Technologies for Military Simulation
}

\author{
Sean Owens, Paul Scerri, Robin Glinton, Bin Yu and Katia Sycara \\ School of Computer Science \\ Carnegie Mellon University \\ Pittsburgh, PA 15213, USA \\ \{owens, pscerri, rglinton, byu, katia\}@cs.cmu.edu
}

\begin{abstract}
To perform large-scale coordination in real-world environments requires that many individually complex technologies come together to form integrated solutions. We present an application where several key technologies are integrated into a unified system via a multiagent infrastructure. We show how the synergistic behavior among heterogeneous technologies results in a significant improvement over the performance of the individual technologies acting alone. Critical extensions were required to the language describing required behavior to allow the pieces to work together. Initial experimental results show system performance on a task of coordinating a military convoy in an adversarial environment was significantly improved when all technologies worked together. However, experiments with a human user in the loop showed that significant advances must still be made before such systems can be fielded in the real-world.
\end{abstract}

\section{Categories and Subject Descriptors}

[Multi Agent Systems]; [Multi Agent Coordination]; [Simulation]

\section{INTRODUCTION}

Autonomous coordination has rapidly progressed over recent years, with significant advances in both specific algorithms[7] and complete approaches to coordination being successfully demonstrated[6]. High bandwidth, widely available, low cost communcation and improvements in algorithm scalability are allowing significant advances in the number of agents able to be efficiently coordinated $[9,11]$. Moreover, advances in artificial intelligence[8] and robotics mean that the robots and agents being coordinated are significantly more individually intelligent than in most previous multiagent systems. This progress is quickly putting important, exciting applications of multiagent technology within reach. However, because there have been relatively few demonstra-

Permission to make digital or hard copies of all or part of this work for personal or classroom use is granted without fee provided that copies are not made or distributed for profit or commercial advantage and that copies bear this notice and the full citation on the first page. To copy otherwise, to republish, to post on servers or to redistribute to lists, requires prior specific permission and/or a fee.

AAMAS'06 May 8-12 2006, Hakodate, Hokkaido, Japan.

Copyright 2006 ACM 1-59593-303-4/06/0005 ...\$5.00. tions of large scale, highly complex multi-agent systems, it is unclear whether existing automated coordination techniques are sufficient for these very complex systems or whether key challenges remain.

Many exciting multi-agent and multi-robot applications have been demonstrated in recent years. Systems including RETSINA-OOA[4] and the Electric Elves[2], have demonstrated coordination of heterogeneous sets of entities to achieve complex objectives. However, in many of these systems, much of the intelligence can be attributed to the coordination, with the individual agents being relatively simple, e.g., web services. Some military simulation environments involve both very complex individual technologies and large numbers of heterogeneous entities, but do not typically make extensive use of state-of-the-art automated coordination technology[1]. Thus, despite the obvious potential for automated coordination of large numbers of intelligent heterogeneous entities, there is a lack of sufficiently high fidelity systems that can expose key remaining challenges. Moreover, in most of these systems effective user control was not required, yet many real-world applications cannot be deployed without such control.

To better understand the challenges of complex coordination of intelligent entities, we have developed a simulation environment, called Sanjaya, where successful performance requires effectively dealing with these type of challenges. Sanjaya is a large scale military simulation where many realistic issues including uncertain sensing, diverse terrain, an intelligent adversary, large numbers of units and complex plans, are modeled. A sophisticated terrain analysis agent[5] provides the team with expected locations of opposing forces and safe paths for travel. A sensor fusion process is available to take sensor readings from multiple sources and reduce uncertainty when distinguishing between opponent and civilian vehicles. Importantly, a human commander has high level control and makes decisions to strike identified opponents. Each of the system components is able to use input from the other components and provide input to those components. Intuitively, synergies should occur in Sanjaya, since the various agents should be able to mutually provide each other with input to improve performance. Thus, evidence of synergies would both show that the coordination was effective and provide support to Xu's result. Our results showed that such synergies were observed. When all of the technologies were brought to bear friendly asset survivability exceeded cases where subsets of, or none of the available technologies were used. 
A variety of distinctly different approaches to coordination exist, developed to meet specific goals and have specific properties. Due to the dynamic nature of the fundamental task of moving and protecting convoys of ground vehicles, we choose to apply teamwork based coordination, since it is designed to be flexible and robust, key requirements for this domain[3]. The specific proxy-based implementation of teamwork that we use executes user designed team oriented plans instantiated at runtime from templates[10]. While team oriented plans have been used extensively in the past, when attempting to apply them to this domain, we encountered two key problems, both related to the production and use of information during coordination. First, the plans did not have sufficient semantic content to allow the proxy to determine where information produced during plan execution should be directed. For example, when the terrain analysis agent generated a least resistance path, it could not determine what to do with the path. Second, some tasks within a plan could not be initiated until other tasks within the plan produced information to completely specify the task, but there was no way of specifying this. For example, a task for protecting a convoy could not be allocated until the path of the convoy was determined. Extensions adding additional semantics, but not changing the declarative nature of the TOP's were implemented.

While automated coordination and intelligent robot and agent technology is rapidly advancing, human intelligence is staying constant. Several previous investigations have shown that human interaction with intelligent distributed systems is extremely difficult, sometimes even leading to poorer performance than the system on its own[12]. A key interface design issue is how much of the underlying intelligence to make opaque to the user and how much to make transparent. In Sanjaya, we took the approach that when the details of the intelligence reasoning were not critical to the human's reasoning, they were made completely opaque. We performed an initial set of user tests with some users being in control of a system with all the intelligent agents performing and another set of users using a less intelligent system with the same interface. Our results showed that users actually performed relatively better with a less intelligent system, indicating that significant advances need to be made before users can exert effective control over very complex multiagent systems.

\section{SANJAYA}

Sanjaya is a constructive simulation supporting simulation of ground, air and unmanned aerial vehicles. The scenario being tested consists of five convoys of ground vehicles each supported by a group of unmanned aerial vehicles (UAVs), traveling across a $50 \mathrm{~km}$ by $50 \mathrm{~km}$ map defended by 20 opposing tanks. This is a challenging scenario for a number of reasons.

The UAVs have a limited sensing range, and there are not enough of them in the scenario to patrol the entire map. Obviously intelligent coordination is necessary to use the UAVs efficiently. When an opponent has been found the UAVs may be used to attack the oppoent, but in doing so the UAV destroys itself. As we have a limited number of UAVs in the scenario, each UAV used to attack further reduces our ability to sense, and to destroy any further opponents found.

Sanjaya simulates an uncertain sensing environment. Sen- sor readings return a list of possible classifications of sensed objects, with a probability associated with each classification. In the scenario being simulated, sixty trucks, representing civilian noncombatants, are placed on the map and may be confused with enemy forces. UAVs, are a limited resource and must be conserved to attack those entities that we are reasonably certain are actual opposing forces. This means that we must have a reasonably high confidence classification of a sensed entity as a military, non civilian entity before we issue a command to attack it.

Unlike other currently available simulation environments, Sanjaya simulates the kind of uncertainty and confusion that can occur in large scale coordination in real-world environments. We find this type of simulator necessary to study the challenges that large scale real-world coordination poses. Furthermore, this type of simulator is necessary to determine potential synergies that can occur while coordinating complex entities and technologies.

Results illustrate that the infrastructure was able to produce synergies between the individual technologies. However, individual technologies did not always improve overall performance. Importantly for the future deployment of complex multiagent systems, initial user testing showed that users were not able to take full advantage of available technology and often made system performance worse.

\section{REFERENCES}

[1] S. M. Aircraft. The TACSI users guide. technical report GDIO-MI-98:356. Technical report, Saab Military Aircraft, 1995. Edition 5.2.

[2] H. Chalupsky, Y. Gil, C. A. Knoblock, K. Lerman, J. Oh, D. V. Pynadath, T. A. Russ, and M. Tambe. Electric Elves: Agent technology for supporting human organizations. AI Magazine, 23(2), 2002.

[3] P. R. Cohen and H. J. Levesque. Teamwork. Nous, 25(4), 1991.

[4] J. Giampapa and K. Sycara. Team oriented agent coordination in the RETSINA multi-agent system. In Proceedings of Agents02, 2002.

[5] R. Glinton, S. Owens, J. Giampapa, K. Sycara, C. Grindle, and M. Lewis. Terrain based information fusion and inference. In P. Svensson and J. Schubert, editors, Fusion 2004, volume I, Mountain View, CA, Jun 2004. ISIF

[6] M. Goodrich, D. Olsen, J. Crandall, and T. Palmer. Experiments in adjustable autonomy. In H. Hexmoor, C. Castelfranchi, R. Falcone, and M. Cox, editors, Proceedings of IJCAI Workshop on Autonomy, Delegation and Control: Interacting with Intelligent Agents, 2001.

[7] P. J. Modi, W.-M. Shen, M. Tambe, and M. Yokoo. An asynchronous complete method for distributed constraint optimization. In $A A M A S, 2003$.

[8] R. Nair, M. Tambe, and S. Marsella. Role allocation and reallocation in multiagent teams: Towards a practical analysis. In $A A M A S, 2003$.

[9] C. L. Ortiz, R. Vincent, and B. Morisset. Task inference and distributed task management in centibots robotic systems. In $A A M A S, 2005$.

[10] D. Pynadath, M. Tambe, N. Chauvat, and L. Cavedon. Toward team-oriented programming. In Intelligent Agents VI: Agent Theories, Architectures, and Languages, 1999.

[11] P. Scerri, Y. Xu, E. Liao, J. Lai, and K. Sycara. Scaling teamwork to very large teams. In $A A M A S^{\prime} 04,2004$.

[12] N. Schurr, J. Marecki, M. Tambe, P. Scerri, J. Levis, and N. Kasinadhuni. The future of disaster response: Humans working with multiagent teams using DEFACTO. In $A A A I$ Spring Symposium on Homeland Security, 2005. 